\title{
Biología y educación: un nuevo punto de encuentro
}

\author{
Virna Julisa López \\ Escuela de Biología \\ Universidad Nacional Autónoma de Honduras
}

$\bullet \bullet \bullet \bullet$

\section{Resumen}

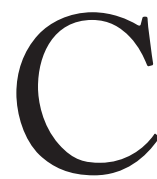

ada día se comprende mejor cómo ocurren las conexiones que tienen lugar en el cerebro humano en diversas actividades. La educación trata de sacar partido de estos nuevos hallazgos para trasladarlos a las aulas de clase. Así surge la neuroeducación, una aproximación que busca mejorar el proceso de enseñanza-aprendizaje desde un enfoque holístico. En ella confluyen las neurociencias, la psicología y la pedagogía. La neurobiología, como rama específica de las neurociencias hace claras aportaciones a la comprensión de cómo ocurre el aprendizaje en el cerebro humano, a través de estudios en el nivel celular y molecular. Es a través del trabajo integrado que ahora se conoce dónde y cómo ocurren diferentes funciones cerebrales tales como la memoria o las emociones.

La neuroeducación busca la creación de un puente que permita que los hallazgos de los laboratorios de neurociencias puedan ser utilizados en las aulas y a su vez, de las aulas se derive información útil para seguir estudiando en los laboratorios. Es esta comunicación la que permitirá el acercamiento hacia un enfoque biopsicosocial de procesos de aprendizaje.
Palabras clave: biología, educación, neuroeducación, neurociencias, aprendizaje.

\section{Abstract}

Every day we have a better understanding of the connections that occur in the human brain during various activities. Education attempts to break down these new discoveries in order to present them in a classroom environment. Thus neuroeducation arises, an approach that seeks to improve the teaching-learning relationship with a holistic focus. In it are other branches of science such as neuroscience, psychology and pedagogy. Neurobiology, as a specific branch of neuroscience makes clear contributions to the understanding of how learning occurs in the human brain, through studies at the cellular and molecular level. Through the integrated work of these branches it is now known where and how different cerebral functions such as memory and emotions occur.

Neuroeducation seeks to create a bridge that will allow findings from neuroscience laboratories to be used in classrooms and in turn, information from the classroom can become useful to continue studying in laboratories. It is this communication that will accomplish a biopsychosocial focus to the learning process.

Keywords: biology, education, neuroeducation, neuroscience, learning.

\section{Introducción}

El conocimiento científico acerca del funcionamiento del cerebro, conocido como neurociencias, avanza rápidamente gracias, en buena medida, a la tecnología de neuroimagen. Esto está permitiendo la comprensión de las conexiones que tienen lugar en el cerebro humano en diversas actividades, cosa que no se podía hacer hace una década. Como resultado se tiene una ola de nuevos puntos de vista acerca de fenómenos como el pensamiento, las emociones, la motivación o el aprendizaje. Estos conocimientos están siendo utilizados en diferentes disciplinas que trascienden la biología y el estudio del cuerpo humano como parte de ella, para incursionar en las ciencias sociales y llevar a la reconsideración de explicaciones ya existentes. Esta incursión es fácil de observar en ciencias como la psicología en la que surgen las neurociencias cognitivas, como también en lo que se conoce como la neurociencia social. Sin embargo, 


\section{Figura 1}

otras disciplinas -como la economía- empiezan a utilizar los resultados de la neurociencia en lo que se conoce como neuroeconomía, o el área de marketing que está desarrollando el novedoso concepto del neuromarketing. Otros espacios disciplinares como la comunicación, las ciencias políticas y la sociología están apenas comenzando a preguntarse si su campo de investigación puede ser informado desde la neurociencia.

La educación por su parte, se encuentra en una posición intermedia en esta escala de disciplinas que han adoptado los resultados de la investigación neurocientífica para llevarlos al desarrollo de su propia área. Así es como surge la neuroeducación, un área multidisciplinar que resulta del traslape de la psicología, la pedagogía y las neurociencias (Figura No.1). El término neuroeducación, neurodidáctica o neuropedagogía surge en 1988 cuando Preiss, propuso introducir una asignatura autónoma en la Universidad de Freiburgo, basada en la investigación cerebral y en la pedagogía. De acuerdo con esta nueva disciplina, la pedagogía escolar y la didáctica general deben conceder más importancia al hecho de que el aprendizaje se basa en procesos cerebrales y que los resultados cognitivos se amplían paralelamente al desarrollo del cerebro infantil. Sobre esa base, la neuroeducación, investiga las condiciones bajo las que el aprendizaje humano puede optimizarse al máximo. (Moncada \& López, 2014)
Neurociencias

(el cerebro y su funcionamento)

para referirnos a la importancia de la biología en el surgimiento de la neuroeducación y las contribuciones que ésta puede hacer hacia un enfoque holístico en el que la educación saque provecho de los nuevos conocimientos que se están generando como resultado del estudio del cerebro.

Pedagogía
(educación y aprendizaie individual)

\section{De la bio-} logía a la pedagogía $\mathrm{La}$ funciones $\mathrm{cog}$ nitivas (y metacognitivas) son consideradas

las facultades mentales más

Figura No.1 La neuroeducación surge del traslape de tres campos disciplinares: la psicología, las neurociencias y la pedagogía, en otras palabras, el estudio de la mente, los fenómenos biológicos que explican el funcionamiento del cerebro y los procesos de formación que tienen lugar a través de

la enseñanza y el aprendizaje. Fuente: elaboración propia

Vale destacar, que las nuevas áreas de estudio están surgiendo gracias a un enfoque multidisciplinar, opuesto a la monidisciplinariedad en la que la ciencia ha trabajado a lo largo de la historia. En la actualidad, la vertiginosa generación de nuevo conocimiento y el cada vez más amplio uso de las tecnologías de la información y comunicación están dejando de manifiesto que la transdisciplinariedad, la multidisciplinariedad y la interdisciplinariedad son una tendencia inevitable para el abordaje sistémico de fenómenos que hasta ahora han sido estudiados de forma aislada. En este artículo hacemos uso de esta tendencia elevadas a la cual se llega a través del aprendizaje. Estas han sido un tema de interés de la psicología y tradicionalmente se han mantenido ajenas a los factores biológicos. Sin embargo, en 1972, dos biólogos, Francisco Varela y Humberto Maturana, proponen el concepto biología del conocimiento como un primer acercamiento entre estas dos disciplinas. Ellos afirman que "el conocimiento, como un proceso de almacenamiento de la información acerca del ambiente y por lo tanto el proceso de vivir, consiste en saber cómo adaptarse a ese ambiente adquiriendo más y más información acerca de su naturaleza" (Maturana \& Varela, 1984, p. XVII).

El conocimiento se adquiere a través de un proceso de aprendizaje y aprender es un rasgo biológico muy primitivo observable aún en especies menores (López, 2013). Aunque el mecanismo que subyace al proceso no está totalmente claro todavía, se sabe que es eminentemente biológico; tal capacidad es necesaria para la sobrevivencia. Kandel (2006) señala que el meca- 
Tabla No.1 Procesos pedagógicos y teorías mentales

Neurobiología

La asociación es reforzada por la repetición.

El "entrenamiento espaciado"favorece la

consolidación.

Hay que evitar interferencia en la consolidación

El control operante favorece el aprendizaje.

El estímulo del contexto promueve la recuperación de

un recuerdo.

El sobre entrenamiento conduce a la formación de hábitos e interfiere con la generalización.
Reglas de Aprendizaje

La repetición es la madre de la sabiduría.

No demasiado en una sola vez (una pausa entre ejercicios es necesaria).

Un poco de calma después de aprender.

Aprender haciendo todo lo que sea posible..

El ambiente de los textos debería ser similar al ambiente

de aprendizaje

El mismo tópico debería ser trabajado de diferentes maneras. nismo celular más simple que permite el aprendizaje y la memoria, ha sido conservado a través de la evolución, por lo tanto ha sido observado y estudiado en organismos primitivos.

Los alcances de la pedagogía están determinados en buena medida por el aprendizaje de contenidos, pro-

34 cedimientos y conductas y es aquí cuando entra en juego la biología como una disciplina de las Neurociencias. En su conjunto, las neurociencias: neurobiología, neurofisiología, neurodesarrollo, neurociencia cognitiva, neurolingüística, neuropsicología, neuropsiquiatría, psicofarmacología, etc. (Cajal, 2006) representan un potencial alternativo para los problemas de la educación. Aunque las neurociencias surgen desde la década de los 60, es en los últimos años que el estudio científico del cerebro adquiere mayor relevancia con el surgimiento de proyectos como "La Década del Cerebro", "Brain" o "El Conectoma Humano". Es así como las neurociencias, a través de la explicación científica de los procesos de aprendizaje, se convierten en una nueva y prometedora área de estudio para responder a las preguntas ¿Cómo es que las personas aprenden?, ¿Cuáles son los mecanismos que explican cómo se da el proceso de aprendizaje?, ¿Qué aspectos limitan a los estudiantes en su aprendizaje?, ¿Cómo se almacena información en la memoria?, ¿Cómo puede recurrirse a la información almacenada cada vez que sea necesario? Este conjunto de disciplinas, aportan al campo pedagógico conocimientos fundamentales acerca de las bases

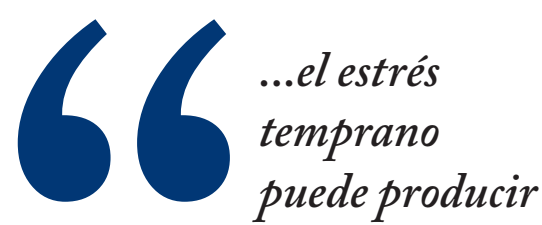
modificaciones epigenéticas duraderas, es decir, cambios estables en la actividad de los genes que determinan el sistema nervioso central y su actividad, así como en el comportamiento...

del aprendizaje, de la memoria, de las emociones y de muchas otras funciones cerebrales que pueden ser estimuladas y fortalecidas en el aula $\mathrm{y}$ de los aspectos que inciden en su desarrollo (Moncada \& López, 2014).

Según Brembs (2009), la neurobio- logía ha permitido la consolidación de algunos procedimientos pedagógicos que se han convertido en reglas, tal como lo resume la Tabla No.1, y que han dado lugar a las conocidas teorías del aprendizaje.

\section{Contribuciones de la} biología

La biología puede contribuir a la comprensión del funcionamiento del cerebro desde una rama específica: la neurobiología. La neurobiología explica el funcionamiento del cerebro desde el nivel celular y molecular, y se complementa con la neuroanatomía y la neurofisiología. De esta manera las evidencias de la función cerebral pueden venir de pruebas de neuroimagen que muestren cambios anatómicos, de la actividad cerebral basada en principios hemodinámicos, o bien de pruebas bioquímicas que muestren los niveles de neurotransmisores o de otras moléculas que participan en la función cerebral. A continuación algunos ejemplos.

Rosenzweig \& Diamond (1972) analizaron la respuesta cerebral ante diferentes estímulos provenientes de ambientes deprivados versus ambientes enriquecidos. Las ratas que se desarrollaron en condiciones ambientales complejas mostraron mayores niveles de factor de 
crecimiento nervioso en comparación con los animales aislados y consecuentemente una mayor dendritización. Mientras Held \& Hein (1963), como muchos etólogos, observaron la conducta en el experimento de los gatos gemelos, cuando trataban demostrar que el aprender a ver estaba asociado al movimiento, este nuevo diseño experimental permitió observar y comparar las neuronas de los ratones de ambos ambientes y de esta manera determinar los cambios morfológicos derivados de estímulos diversos (Figura No. 2).

En el año 2000, Eric Kandel recibió el premio Nobel en Fisiología por su contribución a la comprensión de la transducción de señales en el sistema nervioso, con cuyo trabajo fue posible comprender cómo se forma la memoria de corto y de largo plazo. Sus trabajos, basados en alguna medida en la teoría neuronal propuesta por Ramón y Cajal a finales de 1800, explican el papel que juega la comunicación neuronal en la formación de la memoria.

Otro experimento realizado por Roth y Sweatt (2011) demostró que el estrés temprano puede producir modificaciones epigenéticas duraderas, es decir, cambios estables en la actividad de los genes que determinan el sistema nervioso central y su actividad, así como en el comportamiento. Por ejemplo, los hijos adultos criados por madres que amamantan a sus crías mostraron cambios moleculares en los receptores de glucocorticoides en el hipocampo, en la transcripción del factor de crecimiento neural, de liberación de corticotropina y la sensibilidad de retroalimentación de corticoides.

Una estrategia bioquímica ha permitido identificar la neurotrofina BDNF (Brain Derived Neuroto-
Figura No.2 Experimento con ratas en ambiente deprivado versus am-biente enriquecido

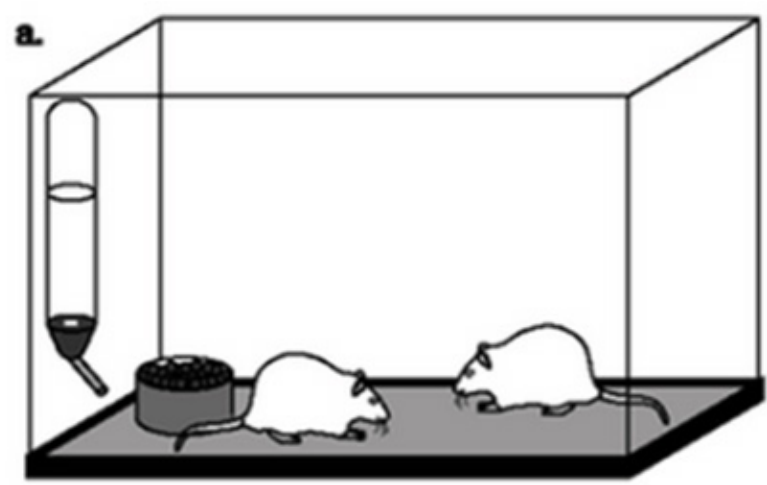

Caja Estándar
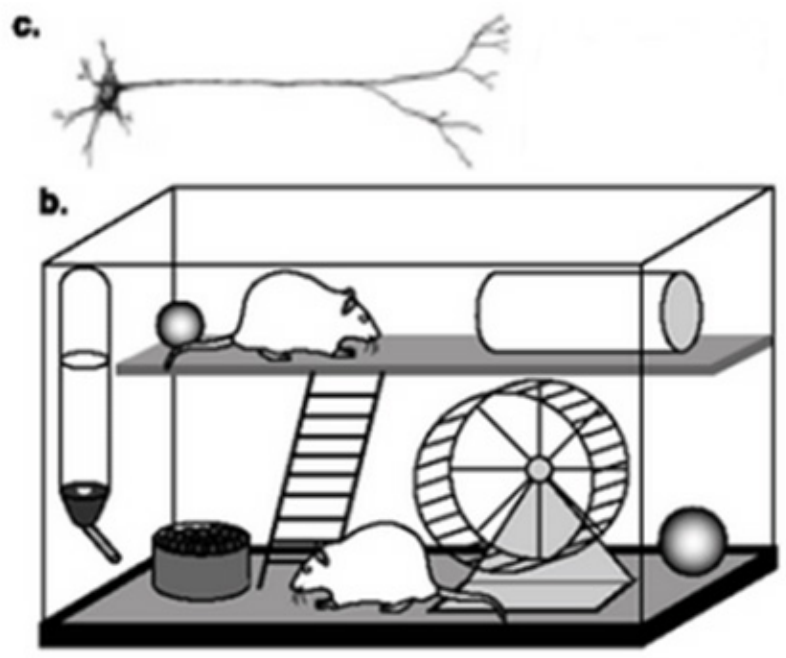

Caja enriquecida

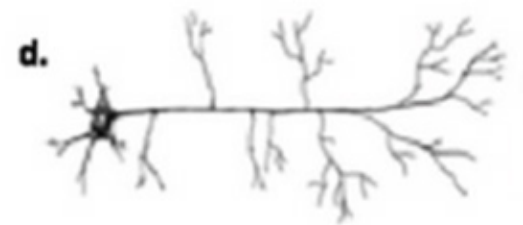

La observación de las neuronas procedentes de ambas ratas demuestra las grandes diferencias en el nivel de dendritización. Las neuronas que provienen de ratas que crecen en un ambiente enriquecido presentan una mayor ramificación que aquellas que no tienen tantos estímulos ambientales.

phic Factor) que pertenece a la familia de proteínas que regulan el crecimiento de la célula nerviosa, como un factor importante para el aprendizaje. Los niveles de BDNF en el cerebro se incrementan con la actividad fisica. Se ha encontrado, en ratones y en humanos, que existe una relación directa entre hacer ejercicios y los niveles de BDNF y a su vez entre los niveles de BDNF y la mejora en el rendimiento de las capacidades cognitivas. A través de los ejercicios y del juego, se desarrollan las funciones ejecutivas y se sintetiza y libera el BDNF. Ambas actividades son cruciales para el aprendizaje y la memoria, activi- 


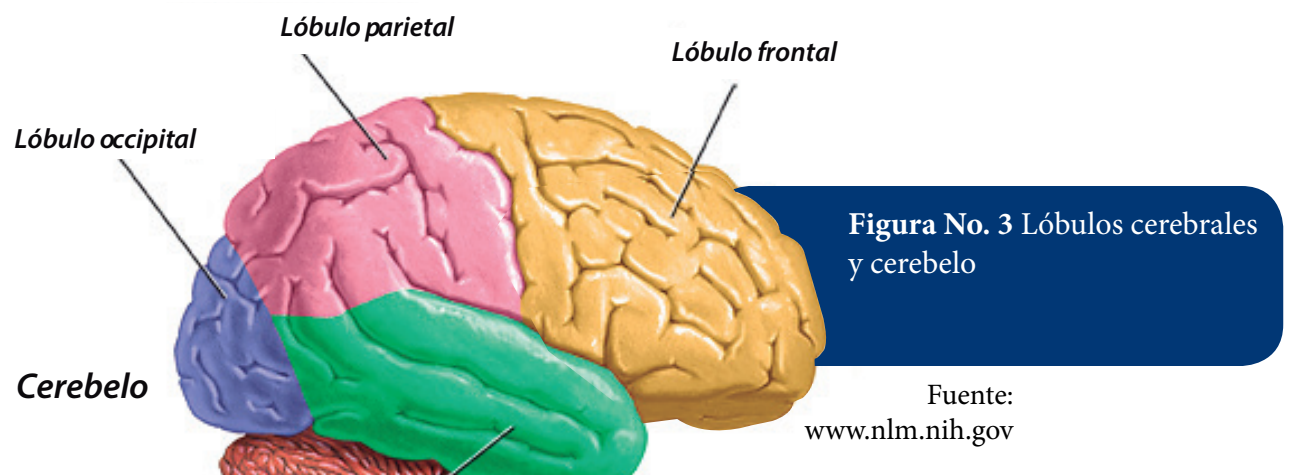

dades que son primariamente importantes en las escuelas donde los estudiantes se tienen que enfocar en tareas específicas, poner atención, pensar críticamente y adquirir nuevo conocimiento y habilidades (Spitzer \& Hollmann, 2013).

Es gracias a este tipo de estudios que en la actualidad se conoce, por

36 ejemplo, cuáles procesos mentales 2014 ocurren en cada parte del cerebro, tal como lo muestra la Tabla No.2, Figuras No.3 y 4.

Aunque a primera vista podría parecer innecesario conocer qué partes del cerebro están implicadas en las diferentes actividades de aprendizaje, este conocimiento puede ayudarnos a entender algunas dificultades de aprendizaje, por un lado, y por el otro, a comprender la interrelación de los procesos que tienen lugar en cada área del cerebro, como por ejemplo, el papel que juegan las emociones en el aprendizaje.

Es de esta manera que es posible estimular el desarrollo de las diferentes regiones y hacerlo tempranamente ahora que se sabe que el mayor desarrollo del cerebro y de todas las conexiones que en él ocurren tienen lugar en la infancia temprana.

Finalmente el advenimiento de las tecnologías de neuroimagen está permitiendo observar el cerebro, en vivo y estudiar su relación con el aprendizaje tanto anatómica como fisiológicamente, de tal forma que se han identificado las partes del cerebro asociadas al aprendizaje de lenguas, música, matemáticas y también a las emociones. Se han estudiado los mecanismos de aprendizaje a corto y

a largo plazo y se sabe que dormir $\mathrm{y}$ hacer ejercicios son actividades que favorecen el aprendizaje. También se sabe que aprender está estrechamente relacionado con la plasticidad del cerebro, una característica observable tanto en su estructura, como en su función. Las neuronas, como unidad básica del sistema nervioso, tienen la capacidad de modificar sus conexiones con otras neuronas y también de desarrollar nuevas conexiones y en esto radica la plasticidad.

Aunque queda mucho por entender en cuanto al aprendizaje y cómo éste ocurre, lo que sí se puede decir actualmente es que aprender tiene lugar a través de la percepción, la memoria y la asociación entre estos dos procesos. Entre estas funciones cognitivas no se puede obviar la importancia de la atención. Con estos procesos involucrados se puede 
Tabla No. 2: Procesos mentales

\begin{tabular}{|c|c|}
\hline Región cerebral & Funciones \\
\hline Lóbulo Frontal & $\begin{array}{l}\text { - Funciones ejecutivas: } \\
\text { Planificar } \\
\text { Razonamiento abstracto } \\
\text { Control de los impulsos } \\
\text { Mantener la atención } \\
\text { - Integra y conecta los componentes de la conducta al más alto nivel. } \\
\text { - Ajuste social de las emociones. } \\
\text { - Conciencia de las habilidades y limitaciones. } \\
\text { - Atención, concentración y lenguaje expresivo están relacionadas con } \\
\text { este lóbulo. }\end{array}$ \\
\hline Lóbulo Parietal & $\begin{array}{l}\text { - Construcción de habilidades y del lenguaje. } \\
\text { - Recuperación de la memoria de largo término. } \\
\text { - Percepción visual y espacial } \\
\text { - Habilidades académicas como la lectura. }\end{array}$ \\
\hline Lóbulo Occipital & $\begin{array}{l}\text { - Función visual } \\
\text { - Percepción y reconocimiento de palabras escritas y así mismo para la } \\
\text { lectura. }\end{array}$ \\
\hline Lóbulo Temporal & $\begin{array}{l}\text { Escuchar, comprender un mensaje y las habilidades de organización y } \\
\text { secuenciación están relacionadas con este lóbulo. } \\
\text { En el lóbulo derecho: } \\
\text { - Habilidades para la música, lenguas extranjeras, memoria visual y } \\
\text { comprensión del entorno. } \\
\text { En el lóbulo izquierdo: } \\
\text { - La producción del lenguaje oral, nombres y la memoria verbal. }\end{array}$ \\
\hline Tallo cerebral & $\begin{array}{l}\text { - Atención básica, excitación y la conciencia. } \\
\text { - Toda la información que proviene del cuerpo y que sale del cerebro } \\
\text { hacia el cuerpo pasa a través del tallo cerebral. }\end{array}$ \\
\hline Cerebelo & $\begin{array}{l}\text { - Coordinación motora relacionada con el movimiento voluntario, el equili- } \\
\text { brio y la memoria de los actos motores reflejos. }\end{array}$ \\
\hline Amígdala & $\begin{array}{l}\text { - Participa en las emociones } \\
\text { - Incrementa el ritmo cardíaco y la presión sanguínea }\end{array}$ \\
\hline Hipocampo & $\begin{array}{l}\text { - Aprendizaje, memoria y emociones. } \\
\text { - Juega un papel importante en la conversión de la memoria de corto plazo } \\
\text { en memoria de largo plazo }\end{array}$ \\
\hline Tálamo & $\begin{array}{l}\text { - Relevo sensorial, recibe señales de los receptores sensitivos, las } \\
\text { procesan y luego las transmiten a las zonas apropiadas de la corteza } \\
\text { sensitiva. }\end{array}$ \\
\hline Hipotálamo & - Conductas de motivación \\
\hline
\end{tabular}


que aprender es un fenómeno corporizado en el cual el cuerpo entero está involucrado a través del sistema nervioso. Consecuentemente, es el estudio del sistema nervioso, principalmente el sistema nervioso central, el que empieza a dar luces acerca de cómo ocurre el aprendizaje.

Comprender cómo tiene lugar el proceso de aprender permitirá aprender a aprender. A partir de esto y de acuerdo a Assman (citado por Gutiérrez, 2010), la pregunta sería: ¿cómo educar y cómo aprender? Estas viejas preguntas involucran nuevas respuestas puesto que el aprendizaje desde el punto de vista biológico es una propiedad que surge de la autoorganización de la vida. Aprender no puede ser reducido a la apropiación de conocimientos ni por los individuos, ni por la humanidad. El aprender es permanente, ocurre a lo largo de la vida y en todas las formas de vida. Aprender es un proceso biológico, todos los seres vivos, para existir, necesitan ser flexibles, adaptarse, reestructurarse, interactuar, crear y coevolucionar, necesitan ser sujetos que aprenden, de otra manera mueren.

\section{Conclusiones}

Configurar el aprendizaje de la forma que mejor encaje en el desarrollo del cerebro es tarea de la neuroeducación y lo logra a la luz de los nuevos conocimientos de la investigación en neurociencias. La neuroeducación posibilita la comprensión de los mecanismos cerebrales que subyacen al aprendizaje, tales como la memoria, el lenguaje, los sistemas sensoriales y motores, la atención, las emociones y el comportamiento, entre otros. Además, ayuda a reconocer los factores de riesgo para el desarrollo cerebral, tales como la desnutrición, las emociones negativas, el alto nivel de stress, el maltrato verbal o físi- co, para citar algunos. Esta información le dará al ámbito educativo mayores posibilidades para reducir o evitar los factores de riesgo, a fin de hacer más saludable y adecuado el entorno de formación.

Una mejora en el procesos enseñanza-aprendizaje en los centros educativos requiere entonces un enfoque holístico, y reconocer que ni la biología, ni la psicología, ni la sociología, pueden mejorar el rendimiento aisladamente; en cambio, es necesario que se integren y se alimenten recíprocamente con los nuevos conocimientos y las nuevas interpretaciones para dar lugar a lo que se considera el enfoque biopsicosocial de la educación.

\section{Referencias}

Brembs, B. (2009). Lernen aus Neurobiologischer Sicht [El aprendizaje desde la perspectiva neurobiológica]. Recuperado de: http://de.slideshare.net/brembs/ lernen-aus-neurobiologischer-sicht

Gutiérrez, F. (2010). Las Nuevas Ciencias de la Vida. Polis, Revista de la Universidad Bolivariana, 9 (25), pp. 223 - 233.

Kandel, E. (2006). In Search of Memory: The Emergence of a New Science of Mind [En busca de la memoria: La aparición de una nueva ciencia de la mente]

. New York: Norton \& Company Inc.

López, V. (2013). Exploring Higher Education in Honduras: a new learning approach with contributions from biology of learning [Exploración de la Educación Superior en Honduras : un nuevo enfoque de aprendizaje con las aportaciones de la biología del aprendizaje ]

- Kaiserslautern: Pädagogischen Materielen.

Moncada, G., \& López, V. (2014).
Educación y Neurociencias: Líneas de Investigación y Políticas Educativas. Tegucigalpa: Inversiones Villamil.

Rosezweig, M., \& Diamond, M. (1972). Brain changes in response to experience [Cambios cerebrales en respuesta a la experiencia]. Scientific American.

Roth, T., \& Sweatt, J. (2011). Epigenetic mechanism and environmental shaping of the brain during sensitive periods of development [Mecanismo epigenético y la conformación del medio ambiente del cerebro durante períodos sensibles de desarrollo]. Journal of CHild Psychology and Psychiatry, 52, 398 - 408.

Spitzer, U., \& Hollmann, W. (2013). Experimental observations of the effects of physical exercise on attention, academic and prosocial performance in school settings [Las observaciones experimentales de los efectos del ejercicio físico sobre la atención el rendimiento, académico y prosocial en el entorno escolar]. Trends in neuroscience and Education, 2, 1-6.:

Recibido: 06/10/2014 Aceptado: 12/10/2014 